\title{
Lipopolysaccharide Delays Closure of the Rat Ductus Arteriosus by Induction of Inducible Nitric Oxide Synthase But Not Prostaglandin E2
}

\author{
Ichige Kajimura, MD; Toru Akaike, MD, PhD; Susumu Minamisawa, MD, PhD
}

\begin{abstract}
Background: The incidence of patent ductus arteriosus is known to be higher in premature neonates with infection than in those without infection. However, the detailed mechanism has not been investigated.

Methods and Results: Lipopolysaccharide (LPS; $100 \mu \mathrm{g} / \mathrm{kg}$ ) was injected into timed-pregnant Wistar rats on day 18 and 19 of pregnancy. The fetuses were delivered by cesarean section on gestational day 21 . Using a rapid wholebody freezing method, it was found that closure of the ductus arteriosus (DA) was significantly delayed in neonates from LPS-injected rats after birth. Histological analysis demonstrated that there was no difference in vascular remodeling of the DA. Quantitative reverse transcriptase-polymerase chain reaction analysis showed that the tumor necrosis factor $a$ and inducible nitric oxide synthase (iNOS) mRNA expression level was significantly increased, but there was no difference in cyclooxygenase 2 and prostaglandin receptor, EP4, mRNA expression in the DA from LPS-injected rats. Moreover, the NOS inhibitor, N $\omega$-Nitro-L-arginine methyl ester hydrochloride, significantly prevented the delayed closure of the DA after birth in neonates from LPS-injected rats.
\end{abstract}

Conclusions: The present study demonstrated that LPS-mediated infection delayed closure of the rat DA without apparent histological changes. iNOS, but not prostaglandin $\mathrm{E}_{2}$, may play a primary role in delayed functional closure of the DA. (Circ J 2016; 80: 703-711)

Key Words: Ductus arteriosus; Infection; Lipopolysaccharide; Nitric oxide

$\mathbf{T}$ he ductus arteriosus (DA) is a fetal bypass vessel between the aorta and the pulmonary artery. Patent DA (PDA) is considered a precursor to mortality and morbidity for especially premature neonates. Every third preterm infant with a birth weight (BW) of 501-1,500 g (very low BW infant) can be expected to have a persistent PDA. ${ }^{1,2}$ Fiftyfive percent of infants whose BW is less than $1,000 \mathrm{~g}$ (extremely low BW infant) have been described to have a symptomatic PDA that leads to medical treatment. ${ }^{1,3,4}$ It has been known that $60-70 \%$ of preterm infants of less than 28 weeks' gestation receive medical or surgical therapy for $\mathrm{PDA}^{5}$ to prevent respiratory decompensation, heart failure, intraventricular hemorrhage, brain injury, chronic lung disease/bronchopulmonary dysplasia, necrotizing enterocolitis and death. ${ }^{1}$ Importantly, the incidence of PDA is known to be even higher in premature neonates with infection than in those without infection. ${ }^{6,7}$ PDA was found in $35.3 \%$ of neonates in a tertiary neonatal intensive care unit who had late-onset sepsis (occurring after 3 days of age) proven by blood culture. ${ }^{8}$ The combined complications of PDA and infection worsen the prognosis of neonates. Duration of ventilator support and hospital stay were longer in neonates with sepsis in the presence of PDA than those in the absence of PDA. ${ }^{8}$ The requirement for additional prescription indomethacin or surgical treatment resulted in an increase in low BW preterm infants when they had a perinatal infection. ${ }^{7}$ Cytokine production by infection may cause PDA because tumor necrosis factor $\alpha(\mathrm{TNF} \alpha)$ is known to be increased in neonates with both infection and PDA. ${ }^{6}$ However, to the best of our knowledge, the mechanism of why infection induces PDA has not been further investigated.

\section{Editorial p 601}

To address this question, it is important to establish an animal model of infection-mediated PDA. Although we and others have previously reported several PDA models such as prostaglandin $\mathrm{E}_{2}\left(\mathrm{PGE}_{2}\right)$-receptor EP4 knockout mice, ${ }^{9,10}$ and Brown-Norway rats, ${ }^{11}$ most of them are congenital types of PDA, but not an infection-induced PDA. Lipopolysaccharide (LPS) has been widely used as an infection model in fetus and neonate. ${ }^{12-14}$ LPS is the major component of the outer membrane of Gram-negative bacteria and it triggers an inflamma-

Received September 30, 2015; revised manuscript received December 17, 2015; accepted December 27, 2015; released online January 27 ,

2016 Time for primary review: 22 days

Department of Cell Physiology, The Jikei University School of Medicine, Tokyo, Japan

Mailing address: Susumu Minamisawa, MD, PhD, Department of Cell Physiology, The Jikei University School of Medicine, Tokyo 105-8461, Japan. E-mail: sminamis@jikei.ac.jp

ISSN-1346-9843 doi:10.1253/circj.CJ-15-1053

All rights are reserved to the Japanese Circulation Society. For permissions, please e-mail: cj@j-circ.or.jp 
tory cascade that results in septic shock. Bustamante et al demonstrated that maternal administration of LPS dilated the fetal DA. ${ }^{15}$ Therefore, it is intriguing to elucidate the effect of LPS on the patency of the DA regarding both functional (vasoconstriction) and anatomical (vascular remodeling) closure. ${ }^{16}$ Functional closure of the DA is promoted by several factors such as an increase in arterial oxygen tension, a decrease in circulating $\mathrm{PGE}_{2}$, and a decrease in nitric oxide (NO) in the luminal endothelium. The DA also exhibits a characteristic structure such as intimal cushion formation (ICF) during development, especially during a perinatal period. We have demonstrated that chronic activation of prostaglandin receptor, EP4, promotes hyaluronic acid (HA)-mediated ICF in the DA. ${ }^{17} \mathrm{HA}$ is produced by HA synthase 2 (HAS2) and HA promotes the migration of ductus smooth muscle cells. ${ }^{17}$ Therefore, the effect of infection on functional and/or anatomical closure of the DA needs to be elucidated. We investigated the mechanism of infection-induced PDA using maternal LPS administration in the present study.

\section{Methods}

\section{Animals}

Timed-pregnant Wistar rats were purchased from Japan SLC, Inc (Shizuoka, Japan). All animals were cared for in compliance with the guidelines of the American Physiological Society. The experiments were approved by the Ethics Committee on Animal Experiments of The Jikei University.

\section{LPS-Injected Rat Model}

In this study, we used LPS to create a model of chorioamnionitis as a severe infection. Pregnant Wistar rats were anesthetized with isoflurane and intraperitoneally injected with LPS $(100 \mu \mathrm{g} / \mathrm{kg})$ on gestational days 18 and 19. For the control group, the same volume of PBS was injected into pregnant rats in the same manner. Rat fetuses were delivered by cesarean section (CS) on embryonic day 21. LPS from Escherichia coli 055: B5 was purchased from Sigma-Aldrich (St. Louis, MO, USA).

\section{Rapid Whole-Body Freezing Method}

To study the in situ morphology and inner diameter of the DA and the main pulmonary artery (MPA), a rapid whole-body freezing method was used, as previously described, with some modifications. ${ }^{18-21}$ The fetuses from LPS- or PBS-injected rats were delivered by CS on embryonic day 21 . The neonates were placed into a warm incubator for 30 or $60 \mathrm{~min}$ and were then rapidly frozen in liquid nitrogen. The frozen neonates were cut on a freezing microtome in the frontal plane, and the inner diameters of the DA and the MPA were measured under a microscope. We evaluated the patency as the ratio of the DA diameter to the MPA diameter.

\section{Histological Analysis}

Tissues from the placenta, DA and aorta were obtained from LPS-injected rat models. Paraffin-embedded blocks containing these tissues were cut into $3 \mu \mathrm{m}$-thick sections and placed on glass slides. To observe polymorphonuclear leukocyte infiltration, the tissue sections were stained with hematoxylin and eosin (HE). To determine the ICF boundary line, tissue sections of the DA and aorta were stained with Elastica van Gieson stain.

\section{Immunohistochemistry}

HA staining was performed by using sections of the DA and the aorta, as previously described. ${ }^{17,22}$ Briefly, the specimens were deparaffinized, rehydrated, incubated for $30 \mathrm{~min}$ in a peroxidase blocking regent to inactivate endogenous peroxidases, and incubated for $30 \mathrm{~min}$ with $1 \%$ bovine serum albumin with PBS-T to avoid non-specific staining. Tissue sections were incubated with biotinylated HA-binding protein (United States Biological, Salem, MA: $8 \mu \mathrm{g} / \mathrm{ml}$ ) at room temperature for $2 \mathrm{~h}$. The slides were sequentially incubated for $30 \mathrm{~min}$ with a biotinylated secondary antibody, incubated for $30 \mathrm{~min}$ with VECTASTAIN Elite ABC reagent (Vector Laboratories, Burlingame, CA, USA) and incubated for $5 \mathrm{~min}$ with DAB Peroxidase Substrate (Vector Laboratories). The sections were rinsed and stained with hematoxylin and mounted.

\section{Quantitative Reverse Transcriptase (RT)-Polymerase Chain Reaction (PCR) Analysis}

Total RNA was isolated from pooled DA and aorta tissue from neonates from LPS- or PBS-injected pregnant rats at birth using TRI Reagent (Molecular Research Center, Inc, Cincinnati, $\mathrm{OH}$, USA). cDNA was generated using PrimeScript ${ }^{\mathrm{TM}}$ RT Master Mix (Perfect Real Time, Takara, Otsu, Japan). RTPCR analysis was performed using SYBR ${ }^{\circledR}$ Premix Ex Taq ${ }^{\mathrm{TM}}$ II (Tli RNaseH Plus), Bulk (Takara). After initial denaturation at $95^{\circ} \mathrm{C}$ for $30 \mathrm{~s}$, the PCR cycle consisted of denaturation at $95^{\circ} \mathrm{C}$ for $5 \mathrm{~s}$ and annealing and elongation for $60^{\circ} \mathrm{C}$ for $45 \mathrm{~s}$; 45 cycles was performed. The sequences of primers for TNF $\alpha,{ }^{23}$ interleukin-6 (IL-6), ${ }^{24}$ inducible nitric oxide synthase (iNOS), ${ }^{23}$ endothelial NO synthase (eNOS), cyclooxygenase 2 (COX2), ${ }^{25}$ EP4, HAS2 and Toll-like receptor 4 (TLR4) ${ }^{26}$ are listed in Table S1. The abundance of each gene was determined relative to an internal control using $18 \mathrm{~s}$ ribosomal RNA. The sequences of the $18 \mathrm{~s}$ ribosomal RNA primer are listed on Table S1. For each RT-PCR experiment, which included a RT negative control, we confirmed that there was no artificial amplification in any reaction.

\section{NO Synthase Inhibition}

To investigate the effect of a NOS inhibitor, we injected $N \omega$-Nitro-L-arginine methyl ester hydrochloride (L-NAME) or saline into the fetuses from LPS-injected maternal rats intraperitoneally through the uterine wall during a CS. ${ }^{27,28}$ The neonates were placed into a warm incubator for $30 \mathrm{~min}$ and were rapidly frozen in liquid nitrogen as explained above. L-NAME was purchased from Sigma-Aldrich.

\section{Statistical Analysis}

All data are presented as the mean \pm standard error of the mean (SEM) of independent experiments. Statistical analyses were performed using the Mann-Whitney test for the 2 groups that were not normally distributed. A P value of less than 0.05 was considered statistically significant.

\section{Results}

\section{Maternal Administration of LPS Delayed Closure of the DA in Rat Neonates}

To examine the effect of inflammation on DA closure, we observed the DA after birth using the rapid whole-body freezing method. We injected LPS $(100 \mu \mathrm{g} / \mathrm{kg})$ into 25 pregnant rats at embryonic day 18 and 19. Among them, 23 pregnant rats could survive until a CS on embryonic day 21. All 19 PBS-injected pregnant rats survived. Closure of the DA was significantly delayed in neonates from LPS-injected rats when they were observed 30 and $60 \mathrm{~min}$ after birth (Figures 1A-F). In neonates from PBS-injected control rats, the DA diameter was decreased up to $20 \%$ of the MPA within 30 min after birth and 
A

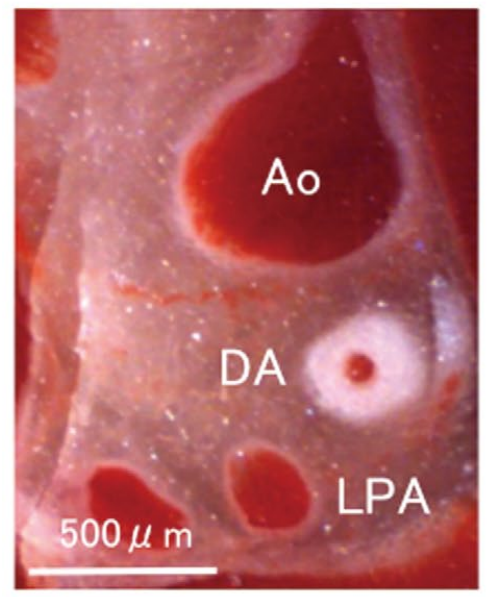

C

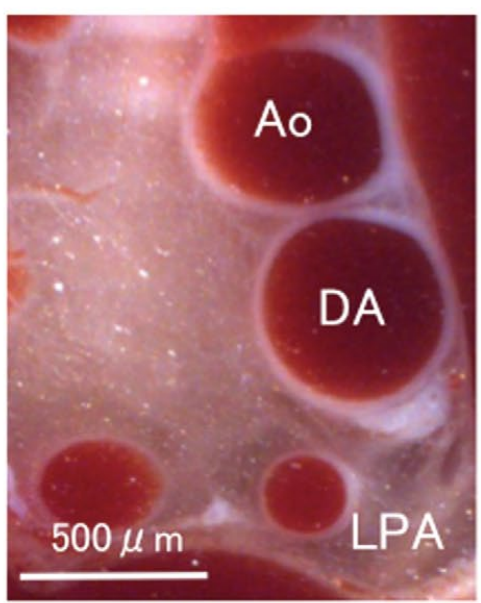

E

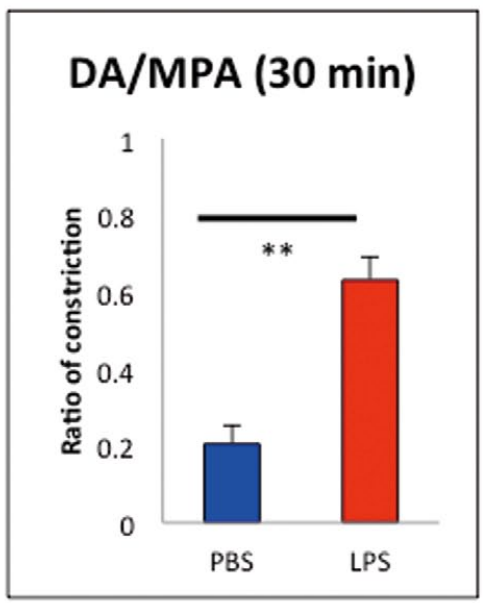

B

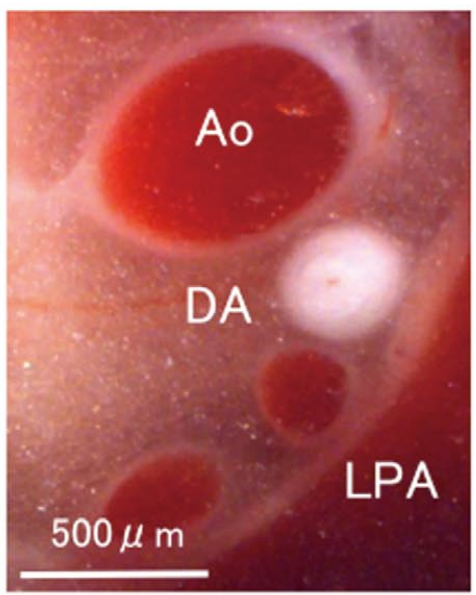

D

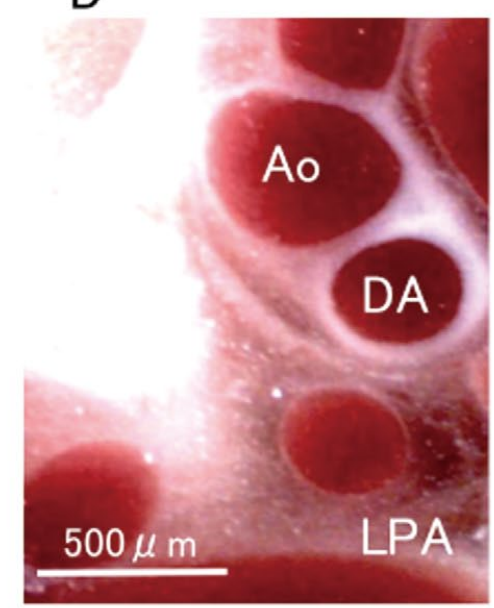

$\mathrm{F}$

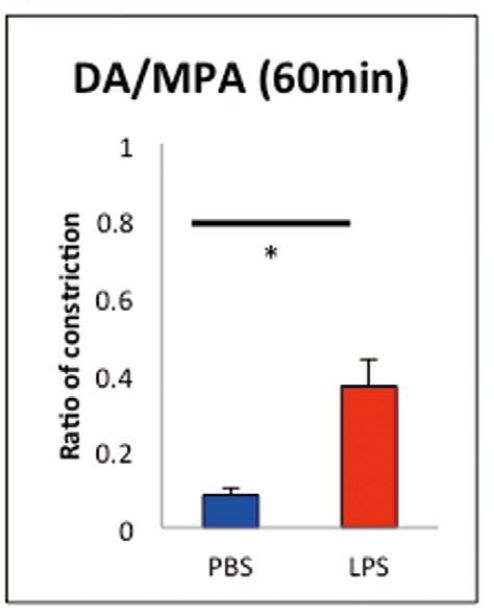

Figure 1. Lipopolysaccharide stimulation delayed rat DA closure. $(\mathbf{A}, \mathbf{B})$ Representative images of a PBS-injected rat sacrificed $30 \mathrm{~min}(\mathbf{A})$ and $60 \mathrm{~min}(\mathbf{B})$ after birth, respectively. (C,D) Representative images of a LPS-injected rat sacrificed $30 \mathrm{~min}$ (C) and $60 \mathrm{~min}$ (D) after birth, respectively. (E,F) LPS stimulation significantly delayed rat DA closure at both $30 \mathrm{~min}(\mathrm{E})$ and $60 \mathrm{~min}(\mathrm{~F})$ after birth. The values are expressed as mean \pm SEM (E, $n=21-34 ; F$, $\mathrm{n}=12-13) .{ }^{*} \mathrm{P}<0.01,{ }^{\star *} \mathrm{P}<0.001$. DA, ductus arteriosus; Ao, aorta; LPA, left pulmonary artery; LPS, lipopolysaccharide; PBS, phosphate buffered saline.
$8 \%$ of the MPA within 60 min after birth. Neonates from LPSinjected rats, however, had a DA diameter that remained up to $63 \%$ of the MPA $30 \mathrm{~min}$ after birth and $37 \%$ of the MPA 60 min after birth (Figures 1E,F).

\section{ICF Developed Normally in the DA of Fetuses From LPS-Injected Rats}

ICF is an essential remodeling process for postnatal closure of the DA. ${ }^{17,29,30} \mathrm{We}$ found that ICF normally developed in the DA of fetuses from both LPS- and PBS-injected rats 


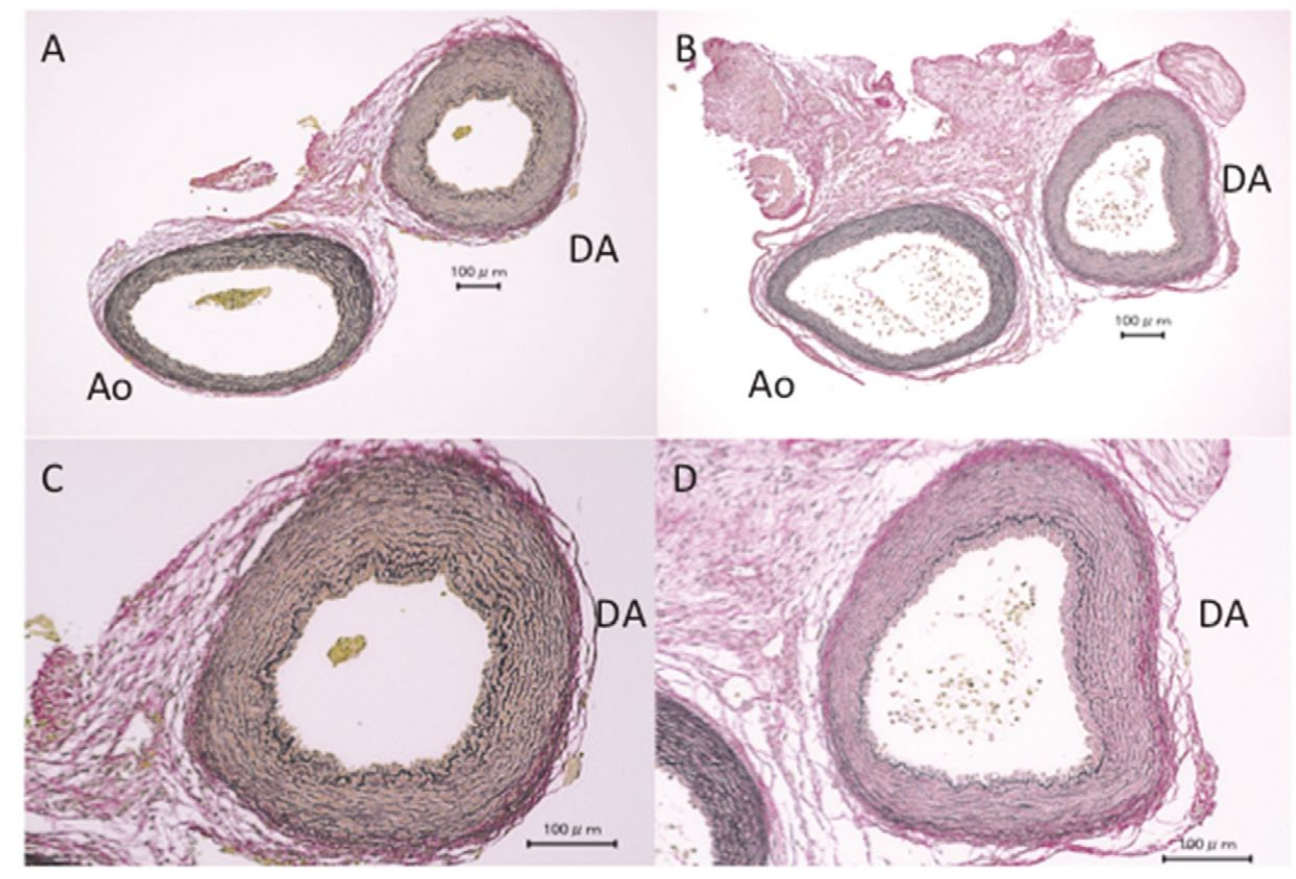

Figure 2. ICF was observed in the DA of LPS-injected rats, which is similar to PBS-injected rats. (A-D) Representative images stained by Elastica van Gieson. (A) PBS-injected DA and Ao. (B) LPS-injected DA. (C,D) Magnified DA from (A) and (B), respectively. ICF was clearly observed in both LPS- and PBS-injected DA $(n=5)$. ICF, intimal cushion formation; LPS, lipopolysaccharide; PBS, phosphate buffered saline; DA, ductus arteriosus; Ao, aorta.

(Figures 2A-D; $\mathrm{n}=5$ ). We did not find evidence of vasculitis such as polymorphonuclear leukocyte infiltration in both LPSand PBS- injected rats.

\section{HA Was Produced Normally in the DA of Neonates From LPS-Injected Rats}

We previously reported that chronic activation of EP4 promoted hyaluronan-mediated ICF. ${ }^{17} \mathrm{HA}$ production was visualized by staining for a HA binding protein (HABP). There was no change in HA production between the DA of the neonates form LPS- and PBS-injected rats (Figures 3A-D; n=5). We examined HAS2 mRNA expression using quantitative RTPCR (Figure 3E; $n=6$ ). There was no significant change in HAS2 mRNA expression between the DA in neonates from LPS- and PBS-injected rats.

\section{Tumor Necrosis Factor $a$, Inducible NO Synthase and TLR4 mRNA Expression Was Increased in the LPS-Stimulated DA} We used quantitative RT-PCR to examine the mechanism of delayed DA closure. Both TNF $\alpha$ and iNOS mRNA expression in the DA and aorta of LPS-injected rats were significantly increased compared with those in the PBS-injected DA and aorta (Figures 4A,C; $n=6$ ). Both IL-6 and eNOS mRNA expression in the DA of LPS-injected rats were not significantly increased (Figures 4B,D; $n=6$ ). Both COX2 and EP4 mRNA expression in the DA and aorta of LPS-injected rats was not significantly different from that of the PBS-injected rats (Figures $4 \mathrm{E}, \mathrm{F} ; \mathrm{n}=6$ ). LPS triggers an inflammatory cascade through TLR4. We found that the expression levels of TLR4 mRNA were increased in the DA and aorta of LPSinjected rats when compared with those in the PBS-injected
DA and aorta, although it did not reach a statistical significance (Figure 4G; $n=6$ ). We examined the expression levels of voltage-gated potassium channels (Kv)1.5 and Kv2.1 mRNAs, because they are known to play important roles in closure of DA. There were no significant change in LPSstimulated DA compared with PBS-injected DA (Figure S1).

\section{L-NAME Inhibited LPS-Induced Patent DA}

When the NOS inhibitor, L-NAME, was injected into the fetuses from LPS-injected maternal rats intraperitoneally through the uterine wall during a CS, the lumen of the DA was significantly decreased up to $17 \%$ of the MPA 30 min after L-NAME injection when compared with the lumen of the DA of saline-injected neonates, which was $31 \%$ of the MPA (Figures 5A-C).

\section{Maternal Administration of Lipolysaccharide Caused Chorioamnionitis}

Macroscopically, the placentas from PBS-injected pregnant rats were lucent and clear (Figure 6A), but the placentas from LPS-injected pregnant rats were dull and dirty (Figure 6B). Histologically, we observed few polymorphonuclear leukocytes on the placentas from PBS-injected pregnant rats (Figures 6C,D), whereas inflammation, indicated by infiltration of polymorphonuclear leukocytes, was extended into the chorion of placentas from LPS-stimulated pregnant rats (Figures $\mathbf{6 E}, \mathbf{F}$; $\mathrm{n}=9-14)$.

\section{Discussion}

Patent DA is considered a precursor to mortality and morbid- 


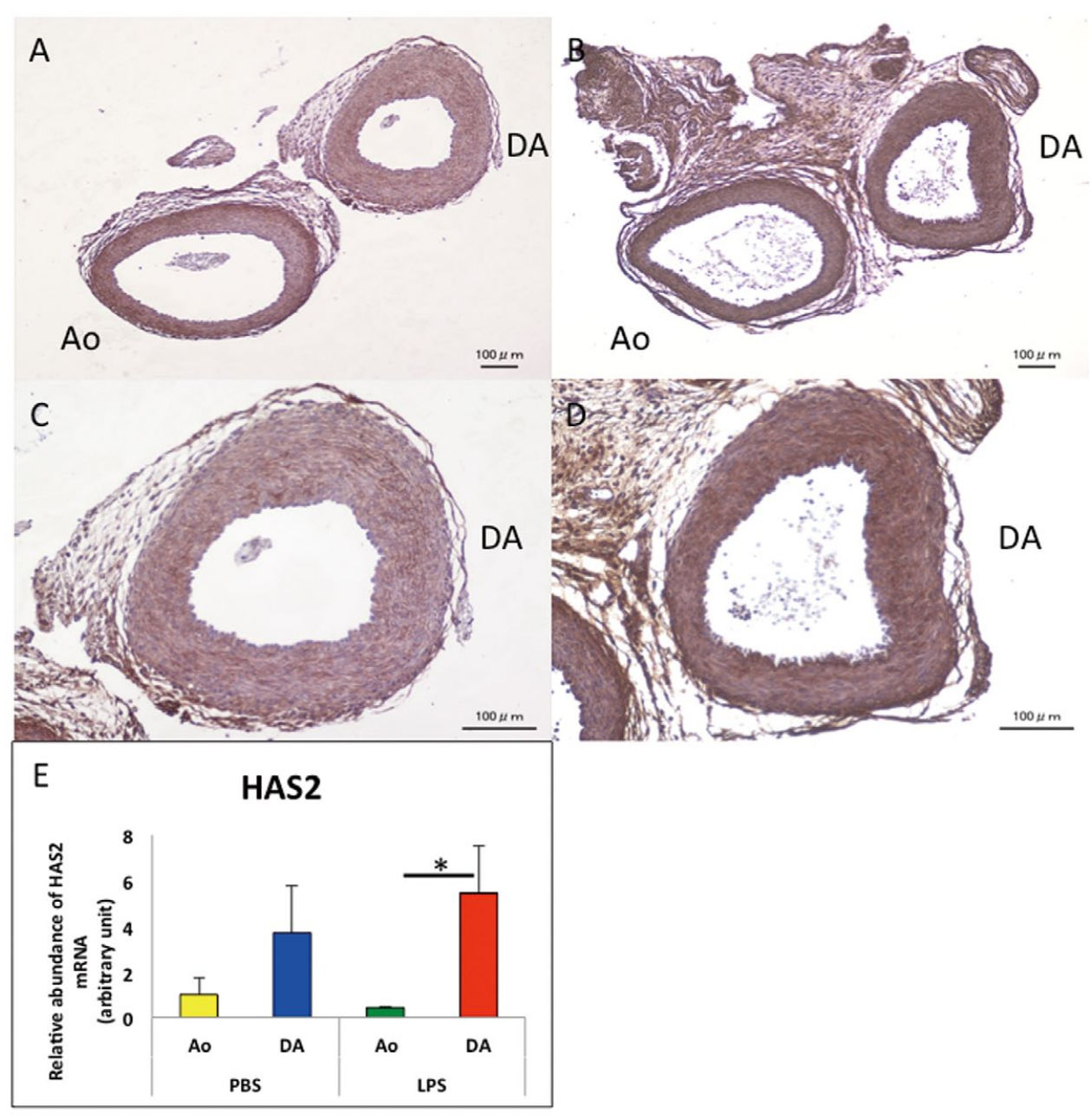

Figure 3. Hyaluronic acid production did not change between DAs stimulated by either LPS or PBS. HA production was visualized by staining for HABP. (A) The DA and aorta from fetuses stimulated by PBS. (B) The DA and aorta from fetuses stimulated by LPS. $(\mathbf{C}, \mathbf{D})$ Magnified DA of $(\mathbf{A})$ and $(\mathbf{B})$. (E) There was no significant change in HAS2 mRNA expression in the DA. The values are expressed as mean \pm SEM. $P<0.05$. ( $A-D, n=5 ; E, n=6$ ). HA, hyaluronic acid; $D A$, ductus arteriosus; Ao, aorta; LPS, lipopolysaccharide; PBS, phosphate buffered saline; HABP, HA binding protein; HAS2, hyaluronic acid synthase 2.

ity in neonates, especially in premature neonates. Importantly, the DA is known to remain open or re-open after birth in neonates with severe infection. ${ }^{6,7}$ However, the mechanism has not been fully understood. To address the problem, we created a stable model by maternal administration of LPS to investigate the mechanism of PDA with infection. ${ }^{12-14}$ In the present study, we demonstrated that maternal administration of LPS delayed closure of the DA by impairing functional closure through $\mathrm{TNF} \alpha$ and iNOS induction. However, the effect of LPS on PDA is controversial. Vucovich et al reported that PDA with infection was caused by aminoglycoside antibiotics, but not by LPS. ${ }^{31}$ In the in vivo experiment by Vucovich et al, mice but not rats were treated, and newborn term gestation mice were injected with LPS at $30 \mathrm{~min}$ of age and then the DA was assessed after a 4-h period of observation. Therefore, the duration of LPS exposure was much shorter in their study than our and Bustamante's experiments, ${ }^{15}$ where chronic inflammatory responses were observed. Thus, we suggest that maternal LPS administration is a suitable method to investi- gate the mechanism of PDA with infection.

The DA from LPS-injected rats did not show any significant histological changes. Closure of the DA depends on 2 mechanisms: functional vasoconstriction and anatomical vascular remodeling. ${ }^{16}$ Maternal rubella infection is known to cause PDA in the fetus that exhibits abnormal anatomical remodeling such as less ICF. ${ }^{32}$ Because we did not find any significant histological changes in the DA of fetuses from LPS-injected rats, the delayed closure of the DA is mainly a result of impaired functional closure of the DA.

Although there were no histological changes in the DA of the fetuses from LPS-injected rats, we found chorioamnionitis in the placenta from LPS-injected rats. Because chorions are parts of the fetus, chorioamnionitis indicates that maternal administration of LPS induced infection in the fetus. Several previous studies have demonstrated that chorioamnionitis is significantly related to PDA, ${ }^{33,34}$ whereas another study did not show a significant relationship between chorioamnionitis and PDA. ${ }^{35}$ Our in vivo study indicates that maternal LPS admin- 


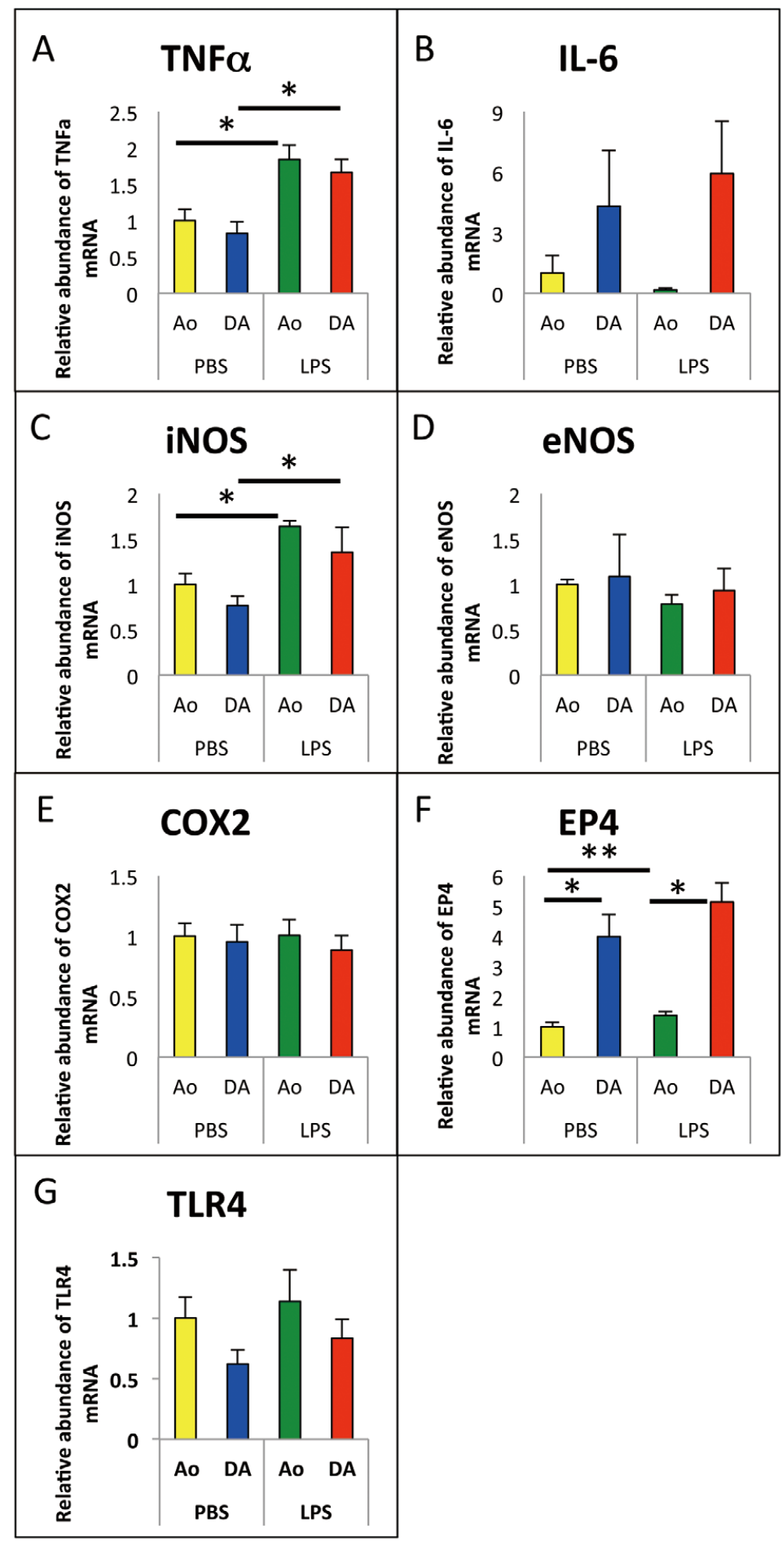

Figure 4. Quantitative RT-PCR shows TNFa, IL-6, iNOS, eNOS, COX2, EP4 and TLR4 mRNA expression in rat DA and Ao. $(\mathbf{A}, \mathbf{C})$ TNFa $(\mathbf{A})$ and iNOS (C) mRNA expression levels in LPS-injected DA and aorta were significantly increased compared with PBS-injected DA and aorta. (B,D) IL-6 (B) and eNOS (D) mRNA expression levels in LPS-injected DA and aorta were not significantly increased compared with PBSinjected DA and aorta. (E,F) COX2 (E) and EP4 (F) mRNA levels in LPS-injected DA and aorta were not significantly increased compared with PBS-injected DA and aorta. (G) TLR4 mRNA levels in LPS-injected DA and aorta tended to increase compared with PBS-injected DA and aorta. The values are expressed as mean \pm SEM. ${ }^{*} P<0.01$, ${ }^{*} \mathrm{P}<0.05(n=6)$. Quantitative RT-PCR, quantitative reverse transcriptase-polymerase chain reaction analyses; TNF $a$, tumor necrosis factor $a$; IL-6, interleukin-6; iNOS, inducible nitric oxide synthase; eNOS, endothelial nitric oxide synthase; COX2, cyclooxygenase 2; EP4, prostaglandin receptor EP4; TLR4, Toll-like receptor 4; DA, ductus arteriosus; Ao, aorta; LPS, lipopolysaccharide; PBS, phosphate buffered saline.

istration caused chorioamnionitis, which then induced inflammatory responses in the fetuses, resulting in PDA.

It is also important to identify the mechanism by which LPS administration impairs functional closure of the DA. LPS triggers an inflammatory cascade, resulting in septic shock in macrophages through TLR4. This response is characterized by production of $\mathrm{TNF} \alpha$ and $\mathrm{NO}$ and a variety of additional inflammatory mediators. ${ }^{36-39}$ LPS also induces $\mathrm{PGE}_{2}$ that regulates TNF $\alpha .{ }^{40}$ In addition, LPS produces iNOS via eNOS and $\mathrm{TNF} \alpha$, which contributes to LPS-induced hypotension. ${ }^{41-44}$ We initially hypothesized that LPS-induced PGE2 caused PDA. However, we found no significant differences in COX2, EP4 
A

B

C

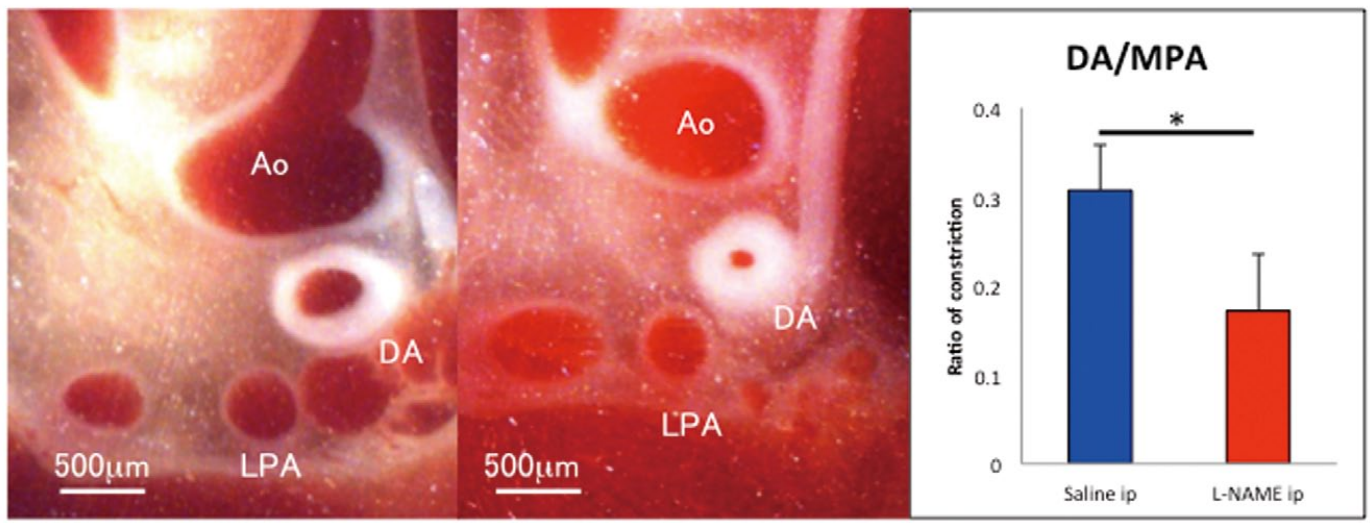

Figure 5. Nitric oxide synthase inhibitor inhibited LPS-induced PDA. (A,B) Representative images of saline-injected neonatal rat (A) and L-NAME-injected neonatal rat (B) 30 min after L-NAME or saline injection. (C) L-NAME injection significantly inhibited LPSinduced PDA. The values are expressed as mean \pm SEM $(C, n=15-16){ }^{*} P<0.05$. DA, ductus arteriosus; Ao, aorta; LPA, left pulmonary artery; LPS, lipopolysaccharide; L-NAME, N $\omega$-Nitro-L-arginine methyl ester hydrochloride; PDA, patent DA.
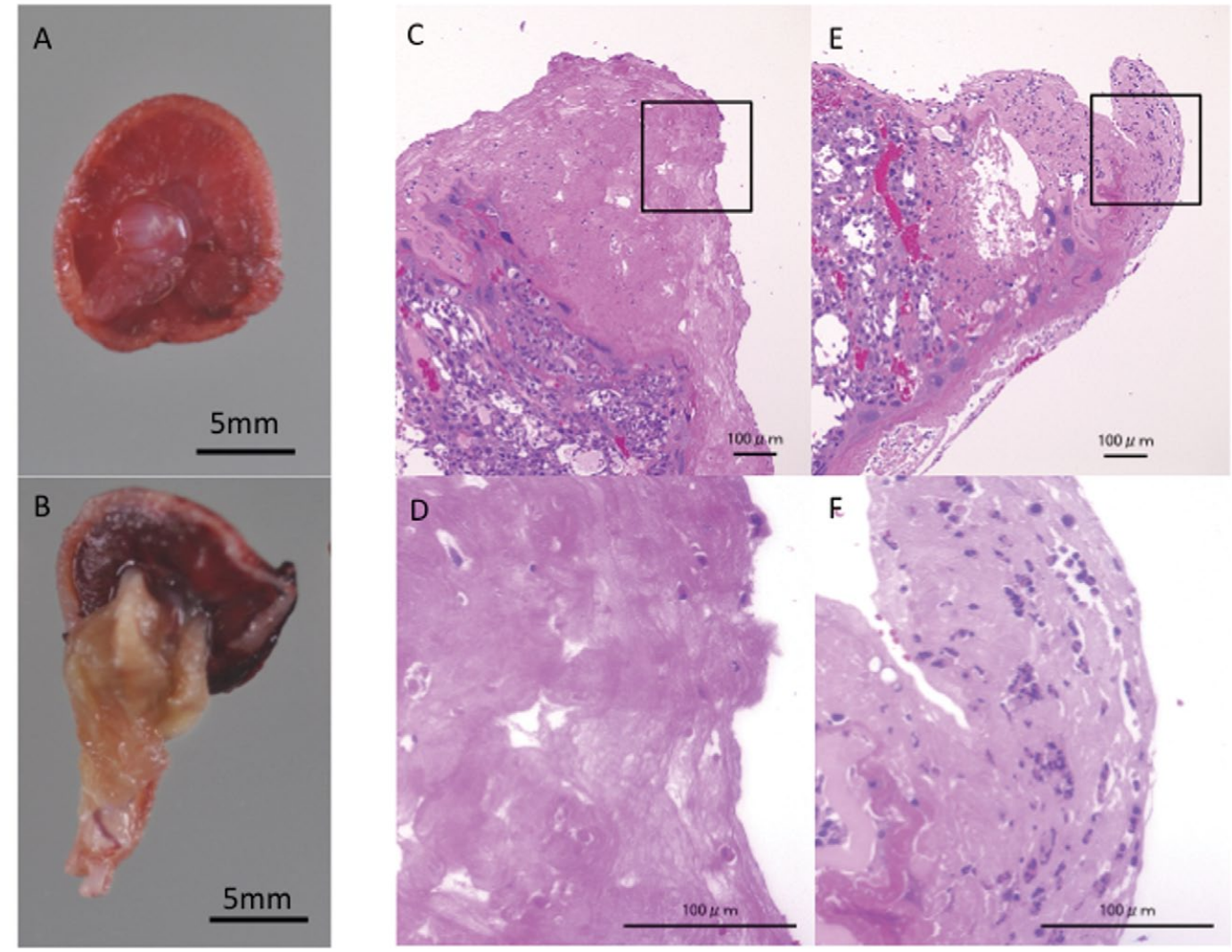

Figure 6. Inflammation was observed in placentas from LPS-injected pregnant rats. (A) A representative placenta from PBSinjected pregnant rats was macroscopically clear and lucent. (B) A representative placenta from LPS-injected pregnant rats was macroscopically dull and dirty. (C,D) Hematoxylin and eosin (HE) staining of the placenta from a PBS-injected pregnant rat. (D) Magnified square from (C). Polymorphonuclear leukocytes were not observed. (E,F) HE staining of the placenta from LPS-injected pregnant rats. (F) Magnified square from (E). Polymorphonuclear leukocytes were observed. Scale bars: $(\mathbf{A}, \mathbf{B}) 5 \mathrm{~mm}$; $(\mathbf{C}-\mathbf{F})$ $100 \mu \mathrm{m}$. LPS, lipopolysaccharide; PBS, phosphate buffered saline. 
and HAS2 mRNA expression and HABP staining in the DA of LPS- and PBS-injected rats, suggesting that the PGE2-EP4 pathway did not contribute to LPS-mediated PDA. Instead, we found that TNF $\alpha$ and iNOS mRNA expression was significantly increased in the maternally LPS-injected DA compared with in the maternally PBS-injected DA. Bustamante et al showed that a NO inhibitor closed the LPS-induced PDA, ${ }^{15}$ which is consistent with the findings of the present study. Therefore, we think that NO plays a primary role in PDA by LPS-induced infection. We showed that the NOS inhibitor, L-NAME, inhibited LPS-induced PDA in mature fetuses. It should be noted that the dilatory effect of NO on the DA is more significant in immature fetuses. ${ }^{45-47}$ Momma and Toyono reported that $\mathrm{NO}$, but not $\mathrm{PGE}_{2}$, played a major role in dilating the DA in preterm fetal rats (embryonic day 19), ${ }^{46}$ and that the importance of NO was relatively decreased during development. ${ }^{46}$ Clyman reviewed that the DA in preterm newborn produced an increased amount of NO after birth although the DA responded to $\mathrm{PGE}_{2}$ persistently. ${ }^{47}$ This report supports the study by Momma and Toyomo. ${ }^{46}$ These findings including the present study suggest that NO inhibitors could be a therapeutic agent to prevent infection-related PDA, especially in immature fetuses. In addition to NO, it has been known that reactive oxygen species $^{48}$ and calpain ${ }^{49}$ are therapeutic targets for inflammation-related vascular diseases. Therefore, further investigation may be required to uncover the role of reactive oxygen species and calpain in inflammation-induced PDA.

In conclusion, maternal administration of LPS delayed closure of the DA by impairing functional closure. This impairment is likely to result from $\mathrm{TNF} \alpha$ and iNOS but not the $\mathrm{PGE}_{2}-\mathrm{EP} 4$ pathway. Our present study suggests that NO inhibition is an effective alternative strategy to COX2 inhibitors for closing the DA when neonates are exposed to severe infection.

\section{Acknowledgments}

We thank Naoko Tomizawa at The Jikei University for technical advice and support.

This work was supported by grants from the Ministry of Education, Culture, Sports, Science and Technology of Japan (S.M., T.A.), the Vehicle Racing Commemorative Foundation (S.M.), The Jikei University Graduate Research Fund (S.M.), The Jikei Research Fund for Graduate Students (I.K.), MEXT-Supported Program for the Strategic Research Foundation at Private Universities (S.M., T.A.), and the Miyata Cardiology Research Promotion Foundation (S.M.).

\section{References}

1. Hamrick SE, Hansmann G. Patent ductus arteriosus of the preterm infant. Pediatrics 2010; 125: 1020-1030.

2. The Vermont-Oxford Trials Network: Very low birth weight outcomes for 1990. Investigators of the Vermont-Oxford Trials Network Database Project. Pediatrics 1993; 91: 540-545.

3. Koch J, Hensley G, Roy L, Brown S, Ramaciotti C, Rosenfeld CR. Prevalence of spontaneous closure of the ductus arteriosus in neonates at a birth weight of 1000 grams or less. Pediatrics 2006; 117: $1113-1121$.

4. Richards J, Johnson A, Fox G, Campbell M. A second course of ibuprofen is effective in the closure of a clinically significant PDA in ELBW infants. Pediatrics 2009; 124: e287-e293, doi:10.1542/ peds.2008-2232.

5. Clyman RI. Ibuprofen and patent ductus arteriosus. $N$ Engl J Med 2000; 343: 728-730.

6. Gonzalez A, Sosenko IR, Chandar J, Hummler H, Claure N, Bancalari E. Influence of infection on patent ductus arteriosus and chronic lung disease in premature infants weighing 1000 grams or less. J Pediatr 1996; 128: 470-478.

7. Pistulli E, Hamiti A, Buba S, Hoxha A, Kelmendi N, Vyshka G. The association between patent ductus arteriosus and perinatal infection in a group of low birth weight preterm infants. Iran J Pediatr 2014; 24: $42-48$.
8. Chiang PJ, Hsu JF, Tsai MH, Lien R, Chiang MC, Huang HR, et al. The impact of patent ductus arteriosus in neonates with late onset sepsis: A retrospective matched-case control study. Pediatr Neonatol 2012; 53: 309-314.

9. Trivedi DB, Sugimoto Y, Loftin CD. Attenuated cyclooxygenase-2 expression contributes to patent ductus arteriosus in preterm mice. Pediatr Res 2006; 60: 669-674

10. Yokoyama U, Minamisawa S, Shioda A, Ishiwata R, Jin MH, Masuda M, et al. Prostaglandin E2 inhibits elastogenesis in the ductus arteriosus via EP4 signaling. Circulation 2014; 129: 487-496.

11. Hsieh YT, Liu NM, Ohmori E, Yokota T, Kajimura I, Akaike T, et al. Transcription profiles of the ductus arteriosus in Brown-Norway rats with irregular elastic fiber formation. Circ J 2014; 78: 12241233.

12. Cai Z, Pan ZL, Pang Y, Evans OB, Rhodes PG. Cytokine induction in fetal rat brains and brain injury in neonatal rats after maternal lipopolysaccharide administration. Pediatr Res 2000; 47: 64-72.

13. Tuzun F, Gencpinar P, Ozbal S, Dilek M, Ergur BU, Duman N, et al. Neuroprotective effect of neotrofin in a neonatal rat model of periventricular leukomalacia. Neurosci Lett 2012; 520: 6-10.

14. Li H, Yuan X, Tang J, Zhang Y. Lipopolysaccharide disrupts the directional persistence of alveolar myofibroblast migration through EGF receptor. Am J Physiol Lung Cell Mol Physiol 2012; 302: L569-L579.

15. Bustamante SA, Pang Y, Romero S, Pierce MR, Voelker CA, Thompson $\mathrm{JH}$, et al. Inducible nitric oxide synthase and the regulation of central vessel caliber in the fetal rat. Circulation 1996; 94: $1948-1953$.

16. Yokoyama U, Minamisawa S, Ishikawa Y. Regulation of vascular tone and remodeling of the ductus arteriosus. J Smooth Muscle Res 2010; 46: 77-87.

17. Yokoyama U, Minamisawa S, Quan H, Ghatak S, Akaike T, SegiNishida E, et al. Chronic activation of the prostaglandin receptor EP4 promotes hyaluronan-mediated neointimal formation in the ductus arteriosus. J Clin Invest 2006; 116: 3026-3034.

18. Momma K, Uemura S, Nishihara S, Ota Y. Dilatation of the ductus arteriosus by prostaglandins and prostaglandin's precursors. Pediatr Res 1980; 14: 1074-1077.

19. Momma K, Toyoshima K, Takeuchi D, Imamura S, Nakanishi T. In vivo reopening of the neonatal ductus arteriosus by a prostanoid EP4-receptor agonist in the rat. Prostaglandins Other Lipid Mediat 2005; 78: 117-128.

20. Yokoyama U, Minamisawa S, Katayama A, Tang T, Suzuki S, Iwatsubo K, et al. Differential regulation of vascular tone and remodeling via stimulation of type 2 and type 6 adenylyl cyclases in the ductus arteriosus. Circ Res 2010; 106: 1882-1892.

21. Yokota T, Aida T, Ichikawa Y, Fujita T, Yokoyama U, Minamisawa $\mathrm{S}$. Low-dose thromboxane $\mathrm{A} 2$ receptor stimulation promotes closure of the rat ductus arteriosus with minimal adverse effects. Pediatr Res 2012; 72: 129-136.

22. Lokeshwar VB, Rubinowicz D, Schroeder GL, Forgacs E, Minna JD, Block NL, et al. Stromal and epithelial expression of tumor markers hyaluronic acid and HYAL1 hyaluronidase in prostate cancer. $J$ Biol Chem 2001; 276: 11922-11932.

23. Paintlia MK, Paintlia AS, Barbosa E, Singh I, Singh AK. N-acetylcysteine prevents endotoxin-induced degeneration of oligodendrocyte progenitors and hypomyelination in developing rat brain. $J$ Neurosci Res 2004; 78: 347-361.

24. Vikman P, Ansar S, Henriksson M, Stenman E, Edvinsson L. Cerebral ischemia induces transcription of inflammatory and extracellular-matrix-related genes in rat cerebral arteries. Exp Brain Res 2007; 183: 499-510.

25. Yin W. Down regulation of COX-2 is involved in hyperbaric oxygen treatment in a rat transient focal cerebral ischemia model. Brain Res 2002; 926: $165-171$.

26. Banerjee A, Abdelmegeed MA, Jang S, Song BJ. Increased sensitivity to binge alcohol-induced gut leakiness and inflammatory liver disease in HIV transgenic rats. PloS One 2015; 10: e0140498, doi:10.1371/journal.pone.0140498.

27. Jackson KE, Jackson DW, Quadri S, Reitzell MJ, Navar LG. Inhibition of heme oxygenase augments tubular sodium reabsorption. Am J Physiol Renal Physiol 2011; 300: F941-F946.

28. Takizawa T, Horikoshi E, Kamata A. Role of the nitric oxide-cGMP system in the regulation of ductus arteriosus patency in fetal rats. $J$ Vet Med Sci 1999; 61: 1277-1280.

29. Rabinovitch M. Cell-extracellular matrix interactions in the ductus arteriosus and perinatal pulmonary circulation. Semin Perinatol 1996; 20: $531-541$.

30. Mason CA, Bigras JL, O'Blenes SB, Zhou B, McIntyre B, Nakamura $\mathrm{N}$, et al. Gene transfer in utero biologically engineers a patent ductus 
arteriosus in lambs by arresting fibronectin-dependent neointimal formation. Nat Med 1999; 5: 176-182.

31. Vucovich MM, Cotton RB, Shelton EL, Goettel JA, Ehinger NJ, Poole SD, et al. Aminoglycoside-mediated relaxation of the ductus arteriosus in sepsis-associated PDA. Am J Physiol Heart Circ Physiol 2014; 307: $\mathrm{H} 732-\mathrm{H} 740$.

32. Gittenberger-de Groot AC, Moulaert AJ, Hitchcock JF. Histology of the persistent ductus arteriosus in cases of congenital rubella. Circulation 1980; 62: 183-186.

33. Arayici S, Kadioglu Simsek G, Oncel MY, Eras Z, Canpolat FE, Oguz SS, et al. The effect of histological chorioamnionitis on the short-term outcome of preterm infants $\leq 32$ weeks: A single-center study. J Matern Fetal Neonatal Med 2014; 27: 1129-1133.

34. Park HW, Choi YS, Kim KS, Kim SN. Chorioamnionitis and patent ductus arteriosus: A systematic review and meta-analysis. PloS One 2015; 10: e0138114, doi:10.1371/journal.pone.0138114.

35. Seliga-Siwecka JP, Kornacka MK. Neonatal outcome of preterm infants born to mothers with abnormal genital tract colonisation and chorioamnionitis: A cohort study. Early Hum Dev 2013; 89: $271-$ 275.

36. Diks SH, van Deventer SJ, Peppelenbosch MP. Lipopolysaccharide recognition, internalisation, signalling and other cellular effects. $J$ Endotoxin Res 2001; 7: 335-348.

37. Lu YC, Yeh WC, Ohashi PS. LPS/TLR4 signal transduction pathway. Cytokine 2008; 42: 145-151.

38. Riedemann NC, Guo RF, Ward PA. Novel strategies for the treatment of sepsis. Nat Med 2003; 9: 517-524.

39. Reis J, Guan XQ, Kisselev AF, Papasian CJ, Qureshi AA, Morrison $\mathrm{DC}$, et al. LPS-induced formation of immunoproteasomes: TNFalpha and nitric oxide production are regulated by altered composition of proteasome-active sites. Cell Biochem Biophys 2011; 60: $77-88$.

40. Kunkel SL, Spengler M, May MA, Spengler R, Larrick J, Remick D. Prostaglandin E2 regulates macrophage-derived tumor necrosis factor gene expression. J Biol Chem 1988; 263: 5380-5384.

41. Thiemermann C, Vane J. Inhibition of nitric oxide synthesis reduces the hypotension induced by bacterial lipopolysaccharides in the rat in vivo. Eur J Pharmacol 1990; 182: 591-595.

42. Vincent JL, Zhang H, Szabo C, Preiser JC. Effects of nitric oxide in septic shock. Am J Respir Crit Care Med 2000; 161: 1781-1785.

43. Connelly L, Madhani M, Hobbs AJ. Resistance to endotoxic shock in endothelial nitric-oxide synthase (eNOS) knock-out mice: A proinflammatory role for eNOS-derived no in vivo. J Biol Chem 2005; 280: $10040-10046$

44. Liao MH, Shih CC, Tsao CM, Chen SJ, Wu CC. RhoA/Rho-kinase and nitric oxide in vascular reactivity in rats with endotoxaemia. PloS One 2013; 8: e56331, doi:10.1371/journal.pone.0056331.

45. Clyman RI, Waleh N, Black SM, Riemer RK, Mauray F, Chen YQ Regulation of ductus arteriosus patency by nitric oxide in fetal lambs: The role of gestation, oxygen tension, and vasa vasorum. Pediatr Res 1998; 43: 633-644.

46. Momma K, Toyono M. The role of nitric oxide in dilating the fetal ductus arteriosus in rats. Pediatr Res 1999; 46: 311-315.

47. Clyman RI. Mechanisms regulating the ductus arteriosus. Biol Neonate 2006; 89: 330-335.

48. Craige SM, Kant S, Keaney JF Jr. Reactive oxygen species in endothelial function from disease to adaptation. Circ J 2015; 79: $1145-$ 1155.

49. Potz BA, Sabe AA, Abid MR, Sellke FW. Calpains and coronary vascular disease. Circ J 2016; 80: 4-10.

\section{Supplementary Files}

Supplementary File 1

Methods

Table S1. The sequences of primers

Figure S1. Quantitative RT-PCR shows (A) voltage-gated potassium channels (Kv) 1.5 and (B) Kv2.1 expression in the rat DA and Ao.

Please find supplementary file(s);

http://dx.doi.org/10.1253/circj.CJ-15-1053 